\title{
Decrease of insoluble glucan formation in Streptococcus mutans by co-cultivation with Enterococcus faecium T7 and glucanase addition
}

\author{
Shin-Hye Yu • So-Hyung Kwak • Thi Thanh Hanh Nguyen • Ye-Seul Seo • \\ Chaeri Song $\cdot$ Il Kyoon Mok $\cdot$ Doman Kim (i)
}

Received: 14 September 2017/ Accepted: 16 November 2017/Published online: 21 November 2017

(C) The Author(s) 2017. This article is an open access publication

\begin{abstract}
Objectives To develop preventive canine oral health bio-materials consisting of probiotics and glucanase to reduce insoluble glucan and volatile sulfur compound formation.

Results Co-cultivation of Enterococcus faecium T7 with Streptococcus mutans at inoculation ratio of 3:1 $(\mathrm{v} / \mathrm{v})$ resulted in $25 \%$ reduction in the growth of Streptococcus mutans. Amounts of soluble and insoluble glucans produced by $S$. mutans were decreased to 70 and 55\%, respectively. Insoluble glucan was decreased from $0.6 \mu \mathrm{g} / \mathrm{ml}$ in $S$. mutans culture to $0.03 \mu \mathrm{g} / \mathrm{ml}$ in $S$. mutans co-cultivated with E. faecium $\mathrm{T} 7$ in the presence of Lipomyces starkeyi glucanase. Volatile sulfur compound, a main component of halitosis produced by Fusobacteria nucleatum, was
\end{abstract}

Shin-Hye Yu and So-Hyung Kwak have contributed equally to this work.

Electronic supplementary material The online version of this article (https://doi.org/10.1007/s10529-017-2478-z) contains supplementary material, which is available to authorized users.

S.-H. Yu · S.-H. Kwak · T. T. H. Nguyen ·

Y.-S. Seo · C. Song · I. K. Mok · D. Kim (ه)

Graduate School of International Agricultural Technology and Institutes of Green Bioscience \& Technology, Seoul National University, Pyeongchang-gun,

Gangwon-do 25354, South Korea

e-mail: kimdm@snu.ac.kr decreased by co-cultivating $F$. nucleatum with $E$. faecium.

Conclusion E. faecium and glucanase can be combined as potentially active ingredients of oral care products for pets by reducing plaque-forming bacteria growth and their by-products that cause cavity and periodontal disease.

Keywords Enterococcus faecium - Glucanase · Insoluble glucan $\cdot$ Streptococcus mutans

\section{Introduction}

Periodontal disease is a common problem occurring in companion animals because pets have insufficient dental care routine. They are exposed to diverse microbes. Thus, pet owners use many strategies to improve the oral health of their pets. Treatment for canine oral diseases is usually expensive. In addition, chemical therapy can induce side effects and drug tolerance (Gorrel et al. 2013). Among bacteria that cause periodontal disease, Streptococcus mutans can synthesize insoluble glucan (mutans) using sucrose. Mutans is involved in initial dental plaque formation following colonization of periodontal bacteria (Takahashi and Nyvad 2011). Dental plaque is a biofilm consisting a group of microorganisms embedded in a matrix mainly containing carbohydrates. The glucan is 
composed of $\alpha-(1 \rightarrow 3), \quad \alpha-(1 \rightarrow 4)$, and/or $\alpha-$ $(1 \rightarrow 6)$-D glucosidic linkages (Takahashi and Nyvad 2011). Hydrolysis of these linkages by using enzymes has been used to remove dental plaque (Ryu et al. 2000). Fusobacterium nucleatum is a major producer of halitosis (Krespi et al. 2006) due to production of volatile sulfide compounds (VSCs) such as $\mathrm{H}_{2} \mathrm{~S}$, methyl mercaptan $\left(\mathrm{CH}_{3} \mathrm{SH}\right)$, and dimethyl sulfide $\left[\left(\mathrm{CH}_{3}\right)_{2} \mathrm{~S}\right]$ by bacterial metabolism (Krespi et al. 2006).

Enterococcus faecium belongs to a group of lactic acid bacteria (Franz et al. 2003). It can be isolated from fermented foods such as sausage, cheese, and fermented vegetables (Giraffa 2003). Inhibitory effects of E. faecium on biofilm formation by cariogenic streptococci have been reported (Kumada et al. 2009; Suzuki et al. 2011). Kumada et al. (2009) have reported that culture supernatant from E. faecium can directly inhibit $S$. mutans biofilm formation. Its inhibition activity is associated with inhibition of $E$. faecium bacterial cells on $S$. mutans strains. Suzuki et al. (2011) have shown that E. faecium in dual cultures possesses bacteriostatic or bactericidal activity against $S$. mutans JCM5705, $S$. mutans Xc, and $S$. sorbinus.

Our previous study has revealed that an endoglucanase of Lipomyces starkeyi can inhibit formation of water-insoluble glucan or mutan (Ryu et al. 2000). However, it is currently unclear whether co-cultivation of E. faecium with $S$. mutans in the presence of $L$. starkeyi endo-glucanase could reduce the formation of water-insoluable glucan from S. mutans or the amount of VSCs produced by $F$. nucleatum. Therefore, the objective of this study was to determine the effect of co-cultivation with E. faecium in the presence or absence of $L$. starkeyi endo-glucanase on amounts of VSCs produced by F. nucleatum, growth of S. mutans, and insoluble glucan formation. Results of this study could provide potential preventive materials to improve canine oral health.

\section{Materials and methods}

Bacterial strains and culture conditions

Streptococcus mutans KCTC3067 was obtained from Korean Collection for Type Cultures. F. nucleatum KCOM 1250 was obtained from Korean Collection for
Oral Microbiology (KCOM, Korea). S. mutans was cultured in brain heart infusion media (BHI) containing sucrose $(20 \mathrm{~g} / \mathrm{l})$. F. nucleatum was cultured in BHI media containing yeast extract $(5 \mathrm{~g} / \mathrm{l})$, hemin $(5 \mathrm{mg} / \mathrm{l})$, and vitamin $\mathrm{K}(0.2 \mathrm{mg} / \mathrm{l})$. They were cultured at $37{ }^{\circ} \mathrm{C}$ for 2 days in $80 \%(\mathrm{v} / \mathrm{v}) \mathrm{N}_{2}, 15 \%$ (v/v) $\mathrm{CO}_{2}$, and $5 \%$ (v/ v) $\mathrm{H}_{2}$ in a gas jar (Oxoid Ltd, England) with a paper sachet (Anaero Gen sachet, AN0025, OXOID Ltd, England). Lipomyces starkeyi glucanase (4.4 U dextranase activity/ml and $0.27 \mathrm{U}$ mutanase activity/ml, respectively) was prepared as describe previously (Ryu et al. 2000).

Isolation and identification of microorganism

E. faecium $\mathrm{T} 7$ was isolated from kimchi and incubated at $37{ }^{\circ} \mathrm{C}$ for $48 \mathrm{~h}$ on de Man Rogosa Sharpe (MRS) agar. To identify the strain, $16 \mathrm{~S}$ rRNA analysis was performed using universal primers 27F (5'-AGAGTT TGATCCTGGCTCAG-3') and 1492R (5'-GGTTAC CTTGTTACGACTT-3'). Additional Biolog GEN III micro test was performed for phenotypic analysis as described previously (HarrisBaldwin and Gudmestad 1996). Development of color was observed using a micro-plate reader at $590 \mathrm{~nm}$ until a similarity index (SIM) was around 0.5. Species identification was made using reference metabolic profiles available in the Biolog GEN III database (version 5.2.1).

Beaker-wire test to determine insoluble glucan formation

Beaker-wire tests were performed as described previously (Chung et al. 2004). Briefly, equal amounts of $S$. mutans and E. faecium $\mathrm{T} 7$ isolates $\left(10^{6} \mathrm{CFU} / \mathrm{ml}\right)$ were co-cultured in a vial containing $10 \mathrm{ml}$ test medium containing a mixture of equal volume of BHI and MRS with $20 \mathrm{~g}$ sucrose/l and $100 \mathrm{mM}$ MOPS (Cutt et al. 2007). Three stainless steel wires $(5 \mathrm{~cm}$ length, $1 \mathrm{~mm}$ diam.) were immersed in each vial and incubated at $37^{\circ} \mathrm{C}$ for $24 \mathrm{~h}$. Each wire was then weighed.

Co-cultivation of E. faecium T7 with S. mutans

To determine the effect of E. faecium T7 co-cultivation on growth of $S$. mutans, culture medium was prepared with the same volume of MRS and BHI media containing sucrose (50 g/l, pH 6.5). Using each seed-culture after overnight growth, $S$. mutans 
$\left(2.8 \times 10^{8} \mathrm{CFU} / \mathrm{ml}\right)$ and $E$. faecium $\mathrm{T} 7$ $\left(8.1 \times 10^{10} \mathrm{CFU} / \mathrm{ml}\right)$ were mixed and inoculated at different ratios [10:0 (S. mutans control), 3:1, 1:1, or $1: 3(\mathrm{v} / \mathrm{v})]$ and incubated at $37{ }^{\circ} \mathrm{C}$ for $12 \mathrm{~h}$ with gentle shaking $(110 \mathrm{rpm})$. Then, we plated serially diluted co-culture broth on BHI agar plates containing $50 \mathrm{~g}$ sucrose $/ 1$ and incubated at $37{ }^{\circ} \mathrm{C}$ for 24 h. S. mutans formed glucans by using sucrose. Therefore, the mucous $S$. mutans colonies were distinguished from E. faecium (Supplementary Fig. 1). Relative survival rate of $S$. mutans was obtained using the following equation:

\section{Relative Survival of S. mutans (\%) \\ $=\frac{\mathrm{CFU} \text { of } S . \text { mutans } \text { coincubated with } E \text {. faecium } \mathrm{T} 7}{\mathrm{CFU} \text { of } S \text {. mutans } \text { control }} \times 100$}

Inhibitory effect of co-cultivation with E. faecium

$\mathrm{T} 7$ on insoluble glucan formation by $S$. mutans

Amounts of soluble and/or insoluble glucan formation and sucrose consumption patterns by $S$. mutans were determined by TLC. After co-cultivation, cell culture was centrifuged at $12,000 \times g$ for $30 \mathrm{~min}$. The TLC plate (silica gel $60 \mathrm{~F}_{254}$ ) was then spotted with $1 \mu \mathrm{l} \mathrm{co-}$ culture supernatant. Culture medium was centrifuged and the pellet was washed twice with distilled water to remove the residual media. After hydrolysis with $1 \mathrm{M}$ $\mathrm{NaOH}, 1 \mu \mathrm{l}$ suspended pellet was spotted onto a TLC plate which was then developed with two ascents of acetonitrile/water $(85: 15, \mathrm{v} / \mathrm{v})$. The developed plate was dried and dipped into $0.3 \%(\mathrm{w} / \mathrm{v}) \mathrm{N}$-(1-naphthyl)ethylenediamine dihydrochloride and $5 \%(\mathrm{v} / \mathrm{v})$ $\mathrm{H}_{2} \mathrm{SO}_{4}$ in methanol followed heating at $120^{\circ} \mathrm{C}$ for $7 \mathrm{~min}$. Concentrations of soluble and/or insoluble glucan or unreacted sucrose were determined as integrated density values using AlphaEaseFC 4.0 image program (Alpha Inotech, CA, USA) with dextran or sucrose as standard as described previously (Mukerjea et al. 1996).

Effect of co-culture of $S$. mutans and E. faecium T7 with additional Lipomyces glucanase on insoluble glucan formation

L. starkeyi glucanase was prepared as described previously (Ryu et al. 2000). Its activity was assayed by incubating the enzyme with $1 \%(\mathrm{w} / \mathrm{v})$ dextran at $30{ }^{\circ} \mathrm{C}$ for various times (Ryu et al. 2000). Standard glucanase assay was performed to determine dextranase activity equivalent using enzyme reaction digest containing $20 \mu \mathrm{l} 1 \%$ dextran, $22 \mu \mathrm{l}$ distilled water, and $0.25 \mu \mathrm{l}$ glucanase. To stop the reaction, $10 \mu \mathrm{l} 1 \mathrm{M} \mathrm{NaOH}$ was added. After adding $148 \mu \mathrm{l}$ copper solution, absorption at $570 \mathrm{~nm}$ was measured to determine the amount of reducing sugar using 96-well plate and a spectrophotometer as described previously (Fox and Robyt 1991). E. faecium was cultured in $51 \mathrm{MRS}$ broth at $37{ }^{\circ} \mathrm{C}$ for $18 \mathrm{~h}$. Cells were centrifuged at $6780 \times g$ for $15 \mathrm{~min}$ and washed several times with distilled water to remove the residual media. Cells were then lyophilized at $-80{ }^{\circ} \mathrm{C}$. After $0.4 \mathrm{~g}$ E. faecium $\mathrm{T} 7\left(10^{9} \mathrm{CFU} / \mathrm{g}\right)$ and $0.2 \mathrm{~g}$ glucanase (22 U dextranase equivalent activity/ml) were mixed in the tube and incubated at room temperature $\left(23^{\circ} \mathrm{C}\right)$ for 2 weeks, cell viability $(\mathrm{CFU} / \mathrm{ml})$ was then determined. Dextranase activity was measured based on the release of reducing sugar from dextran using 3,5dinitrosalicylic acid method (Dols et al. 1997).

Inhibitory effect of E. faecium T7 co-culture on the production of volatile sulfur compounds by $F$. nucleatum

To determine the inhibitory effect of co-culture with E. faecium $\mathrm{T} 7$ on the production of VSCs by $F$. nucleatum, an equal volume of each strain at $10^{9} \mathrm{CFU} /$ $\mathrm{ml}$ was mixed together and vortexed for $10 \mathrm{~s}$ followed by incubation at $37{ }^{\circ} \mathrm{C}$ with gentle shaking (at $110 \mathrm{rpm})$. Then two ml growth medium ( $\mathrm{pH} \mathrm{7)}$ containing $0.1 \%$ (w/v) cysteine, $0.2 \%$ (w/v) $\mathrm{FeSO}_{4}$, and $100 \mathrm{mM}$ MOPS was carefully added into the mixed culture followed by incubation at $37{ }^{\circ} \mathrm{C}$ for $48 \mathrm{~h}$ under anaerobic condition as described previously (Langendijk et al. 1999). $\mathrm{H}_{2} \mathrm{~S}$ production was assessed by determining the degree of appearance of insoluble black iron sulfide $(\mathrm{FeS})$ precipitate in the test tube.

\section{Statistical analysis}

All data are presented as mean \pm standard error of the mean (SEM) from three independent experiments. In each experiment, the test was performed in triplicates. Differences between groups were determined using one-way analysis of variance (ANOVA) followed 
Tukey HSD method. SPSS version 23.0 for Windows (SPSS Inc., Chicago, IL, USA) was used for all statistical analyses. Statistical significance was considered at $p<0.05$. Significantly different insoluble glucan formation was indicated by different superscripts in lower case in Tables.

\section{Results and discussion}

Strain isolation, biochemical characterization, and identification

The 16S rRNA sequence from $\mathrm{T} 7$ strain isolated from kimchi shared 99\% sequence identities with $E$. faecium 16S rRNA sequence (GenBank Accession No: CP006030.1). Based on biochemical characteristics determined with Biolog system using 73 substrate oxidation tests and 21 sensitivity tests (Supplementary Tables 1 and 2), this T7 isolate was identified as $E$. faecium (SIM index: 0.76). E. faecium is commensal in human intestines. It has been used as a probiotic in both animals and human (Franz et al. 2011). Probiotics are live microorganisms that support healthy GI tract. They can also improve health condition of immunity, digestion, and stool quality (Franz et al. 2011).

Inhibitory effect of E. faecium T7 co-cultivation on formation of $S$. mutans insoluble glucan

Based on modified beaker and wire test, the mean weight of artificial biofilm formed on orthodontic wires was $333 \mathrm{mg}$ in the group with $S$. mutans single culture. However, in the co-cultivation group, insoluble glucan was not attached onto the wire (Fig. 1). CFU after co-culture of $S$. mutans/E. faecium T7 at 3:1 (v/v), 1:1 (v/v), or 1:3 (v/v) was decreased to 25,17 , or $14 \%$ of the control (S. mutans single culture), respectively (Fig. 2). E. faecium T7 co-culture also decreased both soluble and insoluble glucans formation in sucrose medium. After co-culture, amounts of soluble glucan and insoluble glucans released to the culture media were decreased to 70 and $55 \%$, respectively, in co-culture of $S$. mutans and E. faecium T7 at inoculation ratio $3: 1(\mathrm{v} / \mathrm{v})$. They were decreased to 53 and $35 \%$, respectively, at inoculation ratio of $1: 1(\mathrm{v} / \mathrm{v})$ and 40 and $17 \%$, respectively, at inoculation ratio of 1:3 (v/v) (Fig. 2).

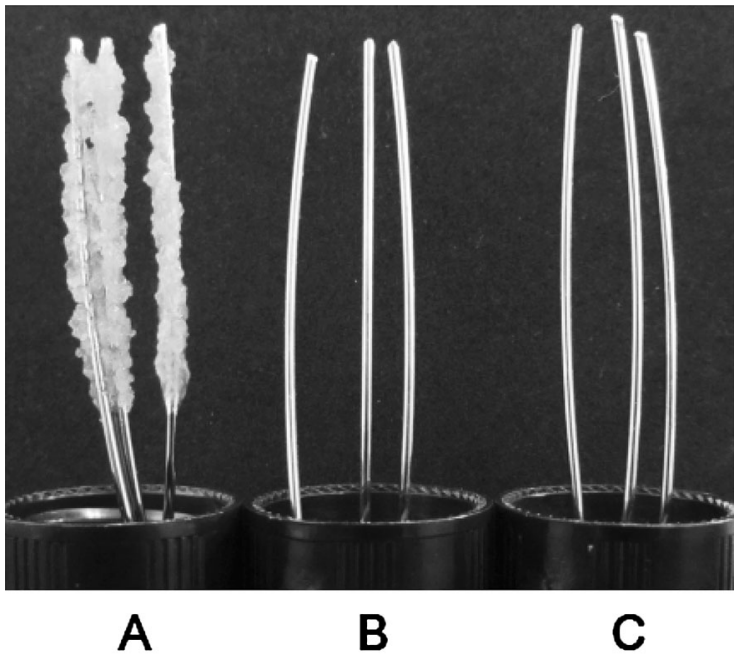

Fig. 1 Effects of E. faecium T7 co-cultivation on amounts of insoluble glucans formed by $S$. mutans on wires using BHI media containing sucrose. (A) Insoluble glucans formed on wires in $S$. mutans single culture, (B) Insoluble glucans formed on wires in E. faecium single culture, (C) Insoluble glucans formed on wires in S. mutans/E. faecium co-culture

Effect of co-culture of S. mutans and E. faecium $\mathrm{T} 7$ with the addition of Lipomyces glucanase on insoluble glucan formation

Equal volumes of MRS and BHI broth media were mixed. The final concentration of sucrose ( $\mathrm{pH} 6.5$ ) was $5 \%(\mathrm{w} / \mathrm{v})$. The final $\mathrm{pH}$ in each cultivation at $25^{\circ} \mathrm{C}$ was 4.6. Two types of product were obtained: soluble dextran from supernatant and insoluble polymer in pellet. With increasing ratio of $E$. faecium added into the co-culutre, less insoluble and soluble glucans were formed. With the addition of glucanase, insoluble dextran formed by $S$. mutans was significantly decreased (Table 1). Amounts of insoluble glucan formed by $S$. mutans culture without the addition of glucanase and with the addition of glucanase were 0.6 and $0.24 \mu \mathrm{g} / \mathrm{ml}$ (61\% reduction), respectively. Under co-cultivation of $S$. mutans and E. faecium at ratio of 3:1 (v/v), amounts of insoluble glucans formed without the addition of glucanase and with the addition of glucanase were, respectively, 0.3 and $0.2 \mu \mathrm{g} / \mathrm{ml}$ (32\% reduction). Under co-cultivation of $S$. mutans and E. faecium at ratio of $1: 1(\mathrm{v} / \mathrm{v})$, amounts of insoluble glucan formed without the addition of glucanase and with the addition of glucanase were 0.25 and $0.03 \mu \mathrm{g} / \mathrm{ml}$ ( $89 \%$ reduction), respectively. Ryu et al. (2000) reported that glucanase from $L$. 
Fig. 2 Relative growth (\%) of $S$. mutans and formation of soluble and insoluble glucans in co-cultures of $S$. mutans and E. faecium T7 at various inoculation ratios $(S$. mutans/E. faecium $\mathrm{T} 7$ at 10:0 (v/v), 3:1 (v/v), 1:1 (v/ $\mathrm{v})$, and $1: 3(\mathrm{v} / \mathrm{v})$

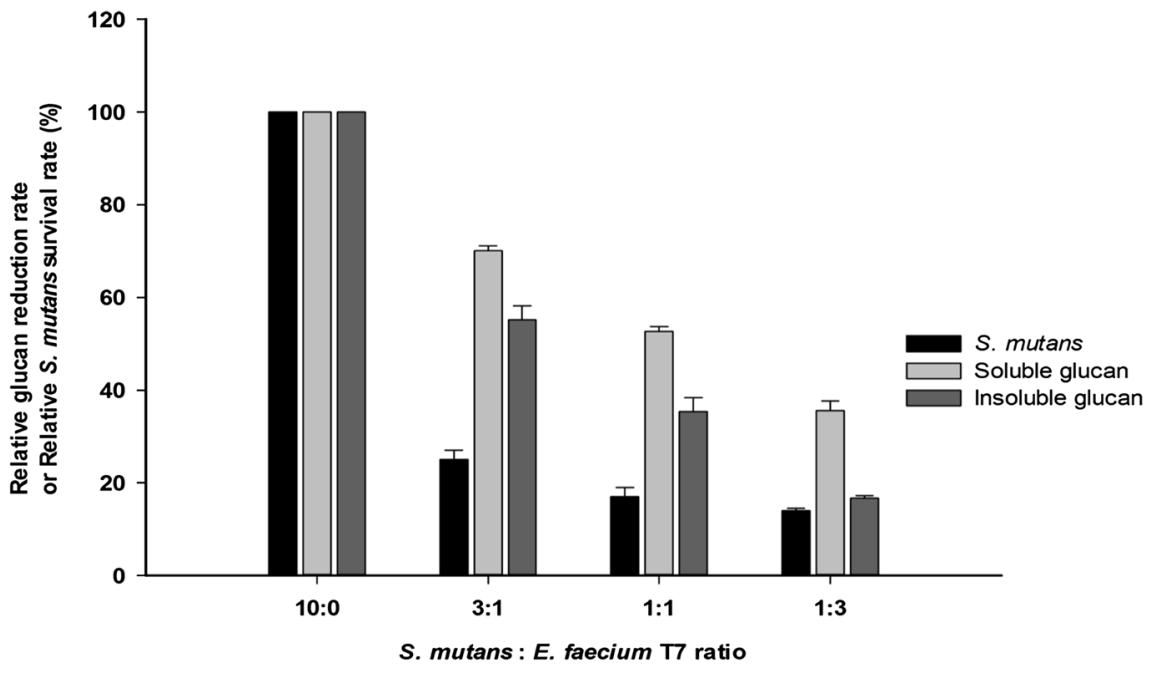

Table 1 Effect of adding glucanase on insoluble glucan formation in S. mutans/E. faecium T7 co-culture

\begin{tabular}{lllll}
\hline Insoluble glucan $(\mu \mathrm{g} / \mathrm{ml})$ & \multicolumn{4}{l}{ S. mutans/E. faecium $\mathrm{T} 7$ ratio $(\mathrm{v} / \mathrm{v})$ co-culture } \\
\cline { 2 - 5 } & $10: 0$ & $3: 1$ & $1: 1$ & $1: 3$ \\
\hline Without glucanase & $0.6 \pm 0.05^{\mathrm{a}}$ & $0.3 \pm 0.04^{\mathrm{b}}$ & $0.25 \pm 0.03^{\mathrm{b}}$ & 0 \\
With glucanase & $0.24 \pm 0.01^{\mathrm{b}}$ & $0.21 \pm 0.03^{\mathrm{b}}$ & $0.03 \pm 0.02^{\mathrm{c}}$ & 0 \\
Reduction $\%$ & 61 & 32 & 89 & - \\
\hline
\end{tabular}

Mean \pm Standard error of the mean (SEM)

${ }^{\mathrm{a}, \mathrm{b}, \mathrm{c}}$ Different superscripts in lower-case letter after values indicate significant difference at $p<0.05$

starkeyi alone can hydrolyze insoluble-glucan of $S$. mutans. Stability of glucanase activity in the presence of E. faecium T7 is shown in Supplementary Table 3. During 14 days of cultutivation at room temperature $\left(25^{\circ} \mathrm{C}\right)$, E. faecium $\mathrm{T} 7$ amounts and glucanase activity were maintained at $10^{9} \mathrm{CFU} / \mathrm{g}$ and $22 \mathrm{U} / \mathrm{g}$ dextranase activity, respectively.

Inhibitory effect of E. faecium $\mathrm{T} 7$ on volatile sulfide compound production in $F$. nucleatum coculture

Halitosis is a common problem in companion animals and humans. It is the first clinical sign of periodontal disease (Culham and Rawlings 1998). Many commercial oral rinses contain anti-plaque and anti-calculus ingredients such as chlorohexidine, cetylpyridinium chloride, alcohol, and/or zinc salts to prevent plaque and halitosis. The most effective and commonly used chemical is chlorhexidine. However, long-term use of chlorhexidine has side effects such as abnormal taste sensation, mucosal irritation, and tooth staining (Robinson 1995). In this study, the effect of E. faecium T7 on the reduction of VSCs by F. nucleatum in coculture was determined by measuring iron sulfide (FeS) formation. E. faecium $\mathrm{T} 7$ inhibited the production of $\mathrm{H}_{2} \mathrm{~S}$ by $\mathrm{F}$. nucleatum in the co-culture, resulting in no black pigment formation in the culture supernatant. However, black pigments were observed in the single culture of $F$. nucleatum (Fig. 3). Thus, cocultivation with $E$. faecium $\mathrm{T} 7$ can reduce the production of VSCs by $F$. nucleatum. It can also reduce the formation of insoluble glucan in sucrose medium produced by S. mutans. Therefore, E. faecium $\mathrm{T} 7$ co-culture can be used as a potentially effective method to improve canine oral health. Its effect can be expanded further by adding glucanase. 


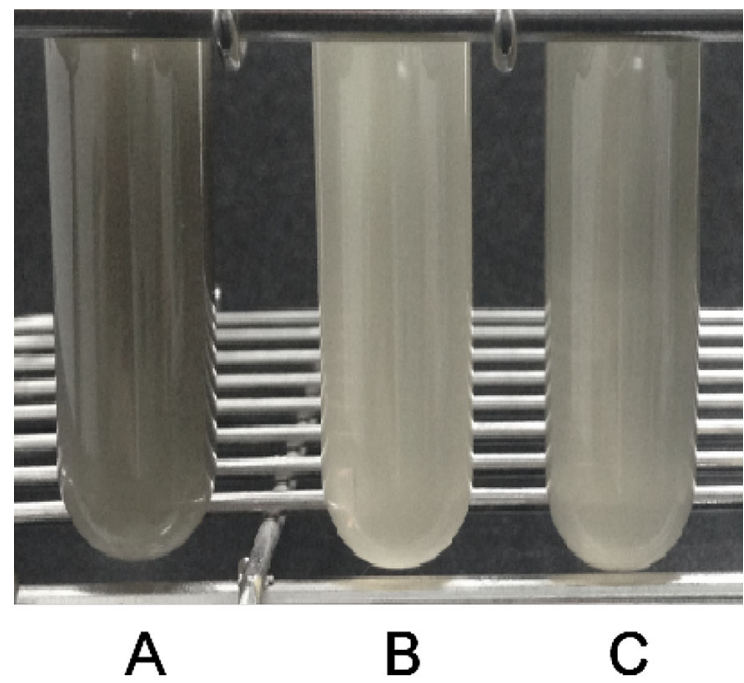

Fig. 3 Effects of E. faecium T7 co-cultivation on the formation of hydrogen sulfide by $F$. nucleatum. (A) $F$. nucleatum single culture, (B) E. faecium single culture, (C) F. nucleatum/E. faecium co-culture. Black pigmentation is a marker for $\mathrm{H}_{2} \mathrm{~S}$ production

\section{Conclusion}

The inhibitory effect of co-cultivation with E. faecium $\mathrm{T} 7$ in the presence of $L$. starkeyi glucanase (containing dextranase and mutanase equivalent activities) on insoluble glucan formation by $S$. mutans has been characterized for the first time. Co-cultivation of $F$. nucleatum with $E$. faecium $\mathrm{T} 7$ also decreased volatile sulfur compound produced by $F$. nucleatum. Therefore, E. faecium and glucanase can be used as potentially active ingredients of oral care products for pets by reducing plaque-forming bacteria growth and their by-products that cause cavity and periodontal disease.

Acknowledgements This work was partially supported by the Basic Science Research Program through the National Research Foundation of Korea (NRF) funded by the Ministry of Education (NRF-2015R1D1A1A01056929; D. Kim, and 2015R1D1A4A0 1020522; T.T. Hanh Nguyen), by Agriculture, Food and Rural Affairs Research Center Support Program, Ministry of Agriculture, Food and Rural Affairs, Republic of Korea, and under the framework of International Cooperation Program managed by the NRF (2016K1A3A1A19945059). Funding was provided by Agriculture, Food and Rural Affairs Research Center Support Program, Ministry of Agriculture, Food and Rural Affairs, Republic of Korea (Grant No. 710002077HD230).
Supporting Information Supplementary Table 1-Biochemical characterization of the T7 isolate for the usage of 71 carbon sources.

Supplementary Table 2-Biochemical characterization of the T7 isolate for the usage of 23 kinds of chemical sensitivity.

Supplementary Table 3-The stability of E. faecium T7 and L. starkeyi mixture at room temperature.

Supplementary Fig. 1-The difference of colonial morphology between $S$. mutans (yellow arrow) and E. faecium (all colonies except yellow arrow) on BHI agar plate containing 50 g sucrose/l.

Open Access This article is distributed under the terms of the Creative Commons Attribution 4.0 International License (http:// creativecommons.org/licenses/by/4.0/), which permits unrestricted use, distribution, and reproduction in any medium, provided you give appropriate credit to the original author(s) and the source, provide a link to the Creative Commons license, and indicate if changes were made.

\section{References}

Chung J, Ha ES, Park HR, Kim S (2004) Isolation and characterization of Lactobacillus species inhibiting the formation of Streptococcus mutans biofilm. Oral Microbiol Immunol 19:214-216

Culham N, Rawlings JM (1998) Oral malodor and its relevance to periodontal disease in the dog. J Vet Dent 15:165-168

Dols M, Remaud-Simeon M, Monsan PF (1997) Dextransucrase production by Leuconostoc mesenteroides NRRL B-1299. Comparison with L. mesenteroides NRRL B-512F. Enzyme Microb Technol 20:523-530

Fox JD, Robyt JF (1991) Miniaturization of three carbohydrate analyses using a microsample plate reader. Anal Biochem 195:93-96

Franz CM, Stiles ME, Schleifer KH, Holzapfel WH (2003) Enterococci in foods-a conundrum for food safety. Int $\mathrm{J}$ Food Microbiol 88:105-122

Franz CM, Huch M, Abriouel H, Holzapfel W, Gálvez A (2011) Enterococci as probiotics and their implications in food safety. Int J Food Microbiol 151:125-140

Giraffa G (2003) Functionality of enterococci in dairy products. Int J Food Microbiol 88:215-222

Gorrel C, Andersson S, Verhaert L (2013) Veterinary dentistry for the general Practitioner2: veterinary dentistry for the general practitioner. Elsevier Health Sciences, New York

HarrisBaldwin A, Gudmestad NC (1996) Identification of phytopathogenic coryneform bacteria using the biolog automated microbial identification system. Plant Dis 80:874-878

Krespi YP, Shrime MG, Kacker A (2006) The relationship between oral malodor and volatile sulfur compound-producing bacteria. Otolaryngol Head Neck Surg 135:671-676

Kumada M, Motegi M, Nakao R, Yonezawa H, Yamamura H, Tagami J, Senpuku H (2009) Inhibiting effects of Enterococcus faecium non-biofilm strain on Streptococcus 
mutans biofilm formation. J Microbiol Immunol Infect 42:188-196

Langendijk PS, Hagemann J, Van Der Hoeven JS (1999) Sulfate-reducing bacteria in periodontal pockets and in healthy oral sites. J Clin Periodontol 26:596-599

Mukerjea R, Kim D, Robyt JF (1996) Simplified and improved methylation analysis of saccharides, using a modified procedure and thin-layer chromatography. Carbohydr Res 292:11-20

Robinson JG (1995) Chlorhexidine gluconate-the solution for dental problems. J Vet Dent 12:29-31
Ryu SJ, Kim D, Ryu HJ, Chiba S, Kimura A, Day DF (2000) Purification and partial characterization of a novel glucanhydrolase from Lipomyces starkeyi KSM 22 and its use for inhibition of insoluble glucan formation. Biosci Biotechnol Biochem 64:223-228

Suzuki N, Yoneda M, Hatano Y, Iwamoto T, Masuo Y, Hirofuji T (2011) Enterococcus faecium WB2000 inhibits biofilm formation by oral cariogenic Streptococci. Int J Dent 2011:834151

Takahashi N, Nyvad B (2011) The role of bacteria in the caries process: ecological perspectives. J Dent Res 90:294-303 